Canadian

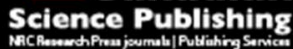

Canadian Geotechnical Journal Revue canadienne de géotechnique

\title{
An analytical approach to assess quality control sample sizes of cement-based solidification/stabilization
}

\begin{tabular}{|r|l|}
\hline Journal: & Canadian Geotechnical Journal \\
\hline Manuscript ID & cgj-2016-0218.R1 \\
\hline Danuscript Type: & Article \\
\hline Complete List of Authors: & $\begin{array}{l}\text { Liza, Rukhsana; Dalhousie University, Civil \& Resource Engineering } \\
\text { Fenton, Gordon; Dalhousie University, Dept. of Engineering Mathematics } \\
\text { Lake, Craig; Dalhousie University, Sexton Campus, } \\
\text { Griffiths, D.; Colorado School of Mines, Civil and Environmental } \\
\text { Engineering }\end{array}$ \\
\hline Keyword: & $\begin{array}{l}\text { hypothesis test errors, solidification, stabilization, sampling, contaminated } \\
\text { soil }\end{array}$ \\
\hline & \multicolumn{2}{|c|}{} \\
\hline
\end{tabular}

SCHOLARONE

Manuscripts 


\section{An Analytical Approach to Assess Quality Control Sample Sizes of Cement- Based Solidification/Stabilization}

Rukhsana Liza ${ }^{1}$, Gordon A. Fenton ${ }^{2}$, Craig B. Lake ${ }^{3}$, and D.V. Griffiths ${ }^{4}$
${ }^{1}$ Department of Civil and Resource Engineering, Dalhousie University, Halifax, Canada: Phone: (519) 760-5493, e-mail: rk962901@dal.ca

${ }^{2}$ Department of Engineering Mathematics, Dalhousie University, Halifax, Canada: Phone: (902) 494-6002, Fax: (902) 423-1801, e-mail: Gordon.Fenton@dal.ca

${ }^{3}$ Department of Civil and Resource Engineering, Dalhousie University, Halifax, Canada: Phone: (902) 494-3220, Fax: (902) 494-3108, e-mail: Craig.Lake@dal.ca

${ }^{4}$ Department of Civil and Environmental Engineering, Colorado School of Mines, Golden, Colorado, USA: Phone: (303) 273-3669, Fax: (303)273-3602, e-mail: D.V. Griffiths@mines.edu 


\title{
An Analytical Approach to Assess Quality Control Sample Sizes of Cement- Based Solidification/Stabilization
}

\author{
Rukhsana Liza, Gordon A. Fenton, Craig B. Lake, and D.V. Griffiths
}

\begin{abstract}
This paper presents an analytical approach to selecting the sample size required to achieve acceptable quality control in a cement-based solidification/stabilization construction cell program intended for the treatment/containment of contaminated soils. The proposed approach is based on the hypothesis test that the cell does not have an acceptably low hydraulic conductivity (the null hypothesis), versus the alternative hypothesis that it does. Analytical solutions are developed to compute the probabilities of both type I (mistakenly rejecting the null hypothesis) and type II (mistakenly failing to reject the null hypothesis) errors as functions of the number of samples and the statistics of the hydraulic conductivity field. The analytical results are validated by Monte Carlo simulations and are then used to develop rational sampling requirements. An example is presented to illustrate how the proposed approach can be used in practice to assess the required sample size for the quality control program of cement-based S/S construction cells.
\end{abstract}

Key words: hypothesis test errors, solidification, stabilization, sampling, contaminated soil, remediation. 


\section{Introduction}

Solidification/stabilization $(\mathrm{S} / \mathrm{S})$ is a source controlled remediation technology for the treatment of contaminated soil (Hills et al. 2015). In cement-based S/S, cement is mixed with the contaminated soil to impart physical and/or chemical changes aimed at minimizing the migration of contaminants from the treated soil into adjacent ground or surface water. For large projects, S/S may be performed at a contaminated site by dividing the entire site into a number of individual cells, which will be referred to as construction cells in this paper, with each cell treated individually with cement. During a quality control (QC) program of cement-based S/S, each construction cell is then "approved" individually, if its hydraulic conductivity is deemed to be acceptable, based on a set of sample data. A construction cell will be accepted if the sample data suggests that the effective hydraulic conductivity $\left(k_{\text {eff }}\right)$ of the cell is below the regulatory value, where the regulatory value $\left(k_{c r i t}\right)$ is as specified by the regulatory agency. The effective hydraulic conductivity of the cell, $k_{e f f}$, is defined as the uniform (spatially constant) hydraulic conductivity value which is equivalent to the actual heterogeneous hydraulic conductivity (which is spatially variable), in terms of the total flow rate through the cell (Fenton and Griffiths 1993). The equation governing the total advective flow rate, $Q$, through a saturated cement-based $\mathrm{S} / \mathrm{S}$ construction cell is given by Darcy's law as follows,

$$
Q=k_{\text {eff }} i A
$$

where $i$ is the hydraulic gradient across the cell and $A$ is the cell area perpendicular to the direction of flow. During QC of cement-based S/S construction cells, samples are collected and tested to estimate the effective hydraulic conductivity. The cell is approved if the estimated effective hydraulic conductivity does not exceed the regulatory value. 
Currently, the sample density method (USACE 2000) is most commonly used to specify the sampling frequency in $\mathrm{S} / \mathrm{S}$ construction cells, requiring a certain number of samples per unit volume. Unfortunately, this method does not adjust for the site variability. Since an increase in the variability of a site increases its randomness, it is logical to believe that the sample density method results in different levels of accuracy in the effective hydraulic conductivity estimate for sites having different variability. In other words, highly variable sites should require more sampling to achieve the same reliability of the approval decision as sites with low variability. This paper aims to develop an analytical approach to select the required sample size for a QC program of cement-based $\mathrm{S} / \mathrm{S}$ construction cells, considering the reliability of the decision about the acceptance or rejection of a construction cell based on the estimated effective hydraulic conductivity.

The overall objective of QC sampling of cement-based S/S construction cells is to ensure, to some acceptable probability level, that the final cell has an effective hydraulic conductivity, $k_{\text {eff }}$, which is less than the regulatory hydraulic conductivity, $k_{c r i t}$. As mentioned previously, the decision about whether a construction cell is acceptable is made on the basis of a set of samples taken from the cell. This decision making process is essentially a hypothesis test where the null hypothesis $\left(H_{0}\right)$ is that the cell is unacceptable, so that the burden of proof is on showing that the alternative hypothesis $\left(H_{a}\right)$ is true, at an appropriate level of confidence.

$$
H_{0}: k_{\text {eff }} \geq k_{\text {crit }}
$$

$$
H_{a}: k_{e f f}<k_{c r i t}
$$

There are two types of errors that may result in making this decision about the acceptability of the cell: 1) a type I error where the sample data suggests that the construction cell is acceptable when it is not, or, 2) a type II error where the sample data fails to suggest that the construction 
cell is acceptable when it actually is acceptable. The aim of this paper is to present analytical solutions for computing these error probabilities (i.e., type I and type II) as a function of the number of samples taken from the S/S construction cell during a QC program.

Random fields are commonly used to model spatially variable engineering properties (Fenton and Griffiths 2008) and will be used here to model the soil hydraulic conductivity field. In this paper, the random hydraulic conductivity field is assumed to be stationary and lognormally distributed. The lognormal assumption of hydraulic conductivity is reasonable for the hydraulic conductivity of S/S sites, as shown by Liza (2014), through statistical analyses of a real site. The lognormally distributed hydraulic conductivity field is characterized by three parameters; its mean, $\mu_{k}$, its coefficient of variation, $v_{k}$, and its correlation length, $\theta_{\ln k}$. The correlation length is a measure of the degree of persistence between hydraulic conductivity values over space and is a parameter of the spatial correlation function, $\rho(\tau)=\exp \left\{-\frac{2|\tau|}{\theta}\right\}$, where $\rho(\tau)$ is the correlation coefficient between two $\ln k$ values separated by distance $\tau$. Smaller values of $\theta_{\ln k}$ imply a rapidly varying field, while larger values of $\theta_{\ln k}$ imply a more slowly varying field. While the mean and standard deviation of the random field can be relatively easily estimated using classical methods (see, e.g., Fenton and Griffiths 2008), estimation of the correlation length is generally not easily done unless an intensive site investigation has been conducted at the site. See Fenton (1999) for a more detailed discussion about the estimation of the correlation length.

In this paper, the random hydraulic conductivity field is assumed to be isotropic and twodimensional as shown in Figure 1.

This two-dimensional assumption is quite reasonable if the layer is thin relative to its planar area, as assumed in this paper. In the two-dimensional model assumed here, the effective hydraulic 
conductivity is assumed to be the local geometric average over the cell area. Fenton and Griffiths (1993), Dagan (1982) and Gutjhar et al. (1978) demonstrated that the geometric average was the best estimate of the effective hydraulic conductivity for relatively square flow regimes. Hence, both the estimated effective hydraulic conductivity, $k_{G}$ and the effective hydraulic conductivity, $k_{\text {eff }}$, of the random field are assumed here to be geometric averages of the sample and pointhydraulic conductivities, respectively. Local averaging reduces the variance of the random field. The final variance depends on the area selected for local averaging, decreasing as the local averaging area increases (Fenton and Griffiths 2008).

Fenton et al. (2015) related the sampling requirements for a QC program of cement-based S/S construction cells to the conductivity field statistics by performing probabilistic simulations. The study examined the influence of correlation length, hydraulic conductivity mean and coefficient of variation on the probabilities of type I and II errors for a specific number of samples taken from a cement-based S/S construction cell during a QC program. Plots provided by Fenton et al. (2015) can be used to estimate the number of samples required to achieve target type I and type II error probabilities. The work presented in this paper is an extension of the work by Fenton et al. (2015). Analytical solutions are developed here to compute the probabilities of type I and II errors as a function of the number of samples taken and the statistics of the hydraulic conductivity field. These analytical solutions enable one to assess the sample size for the QC program of cement-based $\mathrm{S} / \mathrm{S}$ construction cell required to achieve target type I and II error probabilities without the requirement for simulations.

The analytical approximations to the probabilities of type I and II errors will be presented in the next section.

\section{Analytical Solutions for the Probabilities of Type I and Type II Errors}


As mentioned previously, random fields will be used in this paper to model the soil hydraulic conductivity field. The resulting fields can be used to derive the distribution of the effective hydraulic conductivity, conditioned on the samples taken from the field. The conditional distributions of the effective hydraulic conductivity, $k_{\text {eff }}$ and the estimated effective hydraulic conductivity, $k_{G}$ can in turn be determined analytically and used to estimate the probabilities of making type I or II errors in the approval decision process, leading to being able to determine the number of samples required to achieve target error probabilities.

In the site modelling, the two-dimensional field is discretized into $m$ elements (= $m_{x} \times m_{y}$, where $m_{x}$ and $m_{y}$ are the number of elements in the $x$ and $y$ directions, respectively). Each element hydraulic conductivity is assumed to be the geometric average of the hydraulic conductivity over that element and assumed to be lognormally distributed (which it is if $k$ is lognormally distributed, as assumed).

The probabilities of type I and II errors can then be mathematically formulated as follows:

The probability of a type I error, $p_{1}$, is defined as

$$
p_{1}=\mathrm{P}\left[k_{G}<k_{c r i t} \cap k_{e f f}>k_{c r i t}\right\rfloor
$$

while, the probability of a type II error, $p_{2}$, is,

$$
p_{2}=\mathrm{P}\left[k_{G}>k_{\text {crit }} \cap k_{\text {eff }}<k_{\text {crit }}\right\rfloor
$$

where $k_{G}$ and $k_{\text {eff }}$ are geometric averages defined as follows,

$$
k_{G}=\exp \left\{\frac{1}{n} \sum_{j=1}^{n} \ln k_{j}\right\}
$$

$$
k_{e f f}=\exp \left\{\frac{1}{X \times Y} \int_{X \times Y} \ln k(\mathbf{x}) d \mathbf{x}\right\}
$$


In the above,

$n=$ number of samples taken from the random field,

$k_{j}=$ hydraulic conductivity of the $j$ th sampled element of the random field,

$X=$ dimension of the cell in the $x$ direction,

$Y=$ dimension of the cell in the $y$ direction, and

$k(\mathbf{x})=$ hydraulic conductivity at spatial coordinate $\mathbf{x}=\left\{x_{x}, x_{y}\right\}$

In Eq's (3) and (4), both $k_{G}$ and $k_{\text {eff }}$ are lognormally distributed since $k$ is assumed to be lognormal, which means that $\ln k_{G}$, and $\ln k_{\text {eff }}$ are normally distributed with means $\mu_{\ln k_{G}}$ and $\mu_{\ln k_{e f f}}$, respectively, standard deviations $\sigma_{\ln k_{G}}$ and $\sigma_{\ln k_{e f f}}$, respectively, and covariance $\operatorname{Cov}\left[\ln k_{G}, \ln k_{\text {eff }}\right\rfloor$. In turn this means that $\ln k_{G}$ and $\ln k_{\text {eff }}$ follow a bivariate normal distribution, $f_{\ln k_{e f f} \ln k_{G}}(r, s)$, so that the probabilities of type I and II errors become,

$$
\begin{aligned}
& p_{1}=\int_{-\infty}^{\ln k_{\text {crit }}} \int_{\ln k_{c r i t}}^{+\infty} f_{\ln k_{e f f} \ln k_{G}}(r, s) d r d s(7) \\
& p_{2}=\int_{\ln k_{\text {crit }}}^{+\infty} \int_{-\infty}^{\ln k_{c r i t}} f_{\ln k_{e f f} \ln k_{G}}(r, s) d r d s(8)
\end{aligned}
$$

where,

$$
f_{\ln k_{e f f} \ln k_{G}}(r, s)=\frac{1}{2 \pi \sigma_{\ln k_{e f f}} \sigma_{\ln k_{G}} \sqrt{1-\rho^{2}}} \exp \left\{-\frac{1}{2\left(1-\rho^{2}\right)}\left(u^{2}-2 \rho u v+v^{2}\right)\right\}
$$

is the bivariate normal distribution with $u=\left(r-\mu_{\ln k_{e f f}}\right) / \sigma_{\ln k_{e f f}}$, $v=\left(s-\mu_{\ln k_{G}}\right) / \sigma_{\ln k_{G}}$ and $\rho$ is the correlation coefficient between $\ln k_{\text {eff }}$ and $\ln k_{G}$. All other terms are as defined previously. The correlation coefficient, $\rho$ 
and the means and standard deviations of $\ln k_{\text {eff }}$ and $\ln k_{G}$ are defined in

\section{Appendix A.}

There is no closed form solution to the integral of the bivariate normal distribution (not to the univariate normal distribution, for that matter). An approximation proposed by Owen (1959) is used in this study to obtain the probabilities of type I and II errors defined by Eq's (7) and (8), respectively. Let

$$
B(h, w, \rho)=\frac{1}{2 \pi \sqrt{1-\rho^{2}}} \int_{-\infty}^{h} \int_{-\infty}^{w} \exp \left[-\frac{1}{2\left(1-\rho^{2}\right)}\left(u^{2}-2 \rho u v+v^{2}\right)\right] d u d v
$$

where $\rho$ is defined above. An approximation to $B(h, w ; \rho)$ is as follows (Owen 1959)

$$
B(h, w ; \rho)=\frac{1}{2} \Phi(h)+\frac{1}{2} \Phi(w)-T\left(h, a_{h}\right)-T\left(w, a_{w}\right)
$$

if $h w>0$ or if $h w=0$ and $h$ or $w \geq 0$, and

$$
B(h, w ; \rho)=\frac{1}{2} \Phi(h)+\frac{1}{2} \Phi(w)-T\left(h, a_{h}\right)-T\left(w, a_{w}\right)-\frac{1}{2}
$$

if $h w<0$ or if $h w=0$ and $h$ or $w<0$,

where

$$
\begin{gathered}
a_{h}=\frac{w}{h \sqrt{1-\rho^{2}}}-\frac{\rho}{\sqrt{1-\rho^{2}}} \\
a_{w}=\frac{h}{w \sqrt{1-\rho^{2}}}-\frac{\rho}{\sqrt{1-\rho^{2}}} \\
T\left(h, a_{h}\right)=\frac{1}{2 \pi} \int_{0}^{a_{h}} \exp \left[-\frac{1}{2} h^{2}\left(1+u^{2}\right)\right] \\
1+u^{2}
\end{gathered}
$$




$$
T\left(w, a_{w}\right)=\frac{1}{2 \pi} \int_{0}^{a_{w}} \frac{\exp \left[-\frac{1}{2} w^{2}\left(1+v^{2}\right)\right]}{1+v^{2}} d v
$$

and $\Phi$ is the standard normal cumulative distribution function.

Eq's (14a) and (14b) are valid when $a_{h}<1$ and $a_{w}<1$.

When $a_{h}>1, T\left(h, a_{h}\right)$ becomes

$$
T\left(h, a_{h}\right)=\frac{1}{2} \Phi(h)+\frac{1}{2} \Phi\left(h a_{h}\right)-\Phi(h) \Phi\left(h a_{h}\right)-T\left(h a_{h}, \frac{1}{a_{h}}\right)
$$

Similarly, when $a_{w}>1, T\left(w, a_{w}\right)$ becomes

$$
T\left(w, a_{w}\right)=\frac{1}{2} \Phi(w)+\frac{1}{2} \Phi\left(w a_{w}\right)-\Phi(w) \Phi\left(w a_{w}\right)-T\left(w a_{w}, \frac{1}{a_{w}}\right)
$$

$T\left(h a_{h}, \frac{1}{a_{h}}\right)$ in Eq. (14c) can be found by replacing $h$ by $h a_{h}$ and $a_{h}$ by $\frac{1}{a_{h}}$ in Eq. (14a).

Similarly, $T\left(w a_{w}, \frac{1}{a_{w}}\right)$ in Eq. (14d) can be found by replacing $w$ by $w a_{w}$ and $a_{w}$ by $\frac{1}{a_{w}}$ in Eq.

(14b). Eq's (14a) and (14b) are solved in this paper using 16-point Gauss quadrature.

Using Owen's (1959) approximation to the bivariate normal probability, as presented above (Eq's 11 and 12), the probability of a type I error, can be written as

$$
p_{1}=\frac{1}{2} \Phi(h)-\frac{1}{2} \Phi(w)+T\left(h, a_{h}\right)+T\left(w, a_{w}\right)
$$

if $h w>0$ or if $h w=0$ and $h$ or $w \geq 0$, and

$$
p_{1}=\frac{1}{2} \Phi(h)-\frac{1}{2} \Phi(w)+T\left(h, a_{h}\right)+T\left(w, a_{w}\right)+\frac{1}{2}
$$

if $h w<0$ or if $h w=0$ and $h$ or $w<0$

The probability of a type II error is 
[16a]

$$
p_{2}=\frac{1}{2} \Phi(w)-\frac{1}{2} \Phi(h)+T\left(h, a_{h}\right)+T\left(w, a_{w}\right)
$$

if $h w>0$ or if $h w=0$ and $h$ or $w \geq 0$, and

$$
p_{2}=\frac{1}{2} \Phi(w)-\frac{1}{2} \Phi(h)+\mathrm{T}\left(h, a_{h}\right)+\mathrm{T}\left(w, a_{w}\right)+\frac{1}{2}
$$

if $h w<0$ or if $h w=0$ and $h$ or $w<0$

where

$$
\begin{gathered}
h=\frac{\ln k_{c r i t}-\mu_{\ln k_{G}}}{\sigma_{\ln k_{G}}} \\
w=\frac{\ln k_{c r i t}-\mu_{\ln k_{e f f}}}{\sigma_{\ln k_{\text {eff }}}}
\end{gathered}
$$

$a_{h}, a_{w}, T\left(h, a_{h}\right)$, and $T\left(w, a_{w}\right)$ have the same meanings as Eq's (13a), (13b), (14a), and (14b), respectively.

Derivations of Eq's (15) and (16) are presented in Appendix B.

The analysis presented above is for a two-dimensional hydraulic conductivity field as discussed above. If the layer thickness is not small relative to its planar area, the hydraulic conductivity field becomes three-dimensional. In order to extend the analysis to a three-dimensional case, the hydraulic conductivity field should be discretized into $m$ elements $=m_{x} \times m_{y} \times m_{z}$, where $m_{x}$, $m_{y}$ and $m_{z}$ are the number of elements in the $x, y$ and $z$ directions, respectively. Consequently, to find the correlation coefficient, $\rho$ and the means and standard deviations of $\ln k_{\text {eff }}$ and $\ln k_{G}$, the variance reduction function should then be determined over volume, rather than area.

\section{Verification}


The type of probabilistic analyses presented in the previous section can also be performed using simulation programs such as a modified version of the two-dimensional random finite element method (RFEM) program, mrflow2d, presented by Fenton et al. (2015). However, it requires significant time to complete a simulation for a set of statistical parameters. The advantage of the analytical approximations presented in this paper is that they enable one to quickly estimate the probabilities of type I and II errors for a specific number of samples and statistics of the random field. However, the analytical approximations given by Eq's (15) and (16) need to be verified, which is done in this section by comparing to Monte Carlo simulations. Simulations are performed using a modified version of the two-dimensional random finite element method (RFEM) program, mrflow2d, following the method described by Fenton et al. (2015).

For a $20 \mathrm{~m} \times 20 \mathrm{~m}$ random field, discretized into $256\left(m_{x}\right) \times 256\left(m_{y}\right)$ elements, parametric variations considered in the simulations are as presented in Table 1 . These variations cover the 'worst case' conditions of the parameters (Fenton et al. 2015).

The field is sampled at equispaced locations in both directions.

For all parameter sets considered (see Table 1), the probabilities of type I and II errors estimated via simulation are compared to those computed analytically using Eq's (15) and (16), respectively, as illustrated in Figures 2 and 3, respectively. Excellent agreements are obtained between the theory and the simulation (which uses 5000 realizations) for both probabilities of 
type I and II errors for all parameter sets considered, indicating that the proposed analytical solutions can be used to compute the probabilities of type I and type II errors with reasonable confidence. The small discrepancies seen in Figures 2 and 3 are due to natural sampling variation.

\section{Procedure to Select Sample Size}

The analytical solutions presented in this paper to compute the probabilities of type I and II errors (i.e., Eq's (15) and (16), respectively) can be used to estimate the sample size required for the QC program of cement-based S/S construction cell to achieve target type I and II error probabilities. The following steps can be taken to select the sample size, given the desired probabilities of type I and type II errors and the statistics of the random hydraulic conductivity field.

1. For a specified $\mu_{k}$ and $v_{k}$, compute $\sigma_{\ln k}^{2}$ and $\mu_{\ln k}$ using Eq's (A4) and (A3), respectively.

2. Compute $\gamma_{\ln k}(X, Y)=\gamma(X) \gamma(Y)$, where $\gamma(X)$ and $\gamma(Y)$ can be computed using Eq.(A6). Then compute $\mu_{k_{e f f}}$ and $\sigma_{k_{e f f}}$ using Eq's (A1) and (A2), respectively.

3. Compute $\sigma_{\ln k_{e f f}}$ and $\mu_{\ln k_{e f f}}$ using Eq's (A8) and (A7), respectively.

4. Choose a specific sample size and compute $\mu_{\ln k_{G}}$ and $\sigma_{\ln k_{G}}$ using Eq's (A9) and (A10), respectively. Computation of $\sigma_{\ln k_{G}}$ requires computations of the variance reduction function over the element, $\gamma_{\ln k}(\Delta x, \Delta y)=\gamma(\Delta x) \gamma(\Delta y)$, where $\Delta x$ and $\Delta y$ are the 
dimensions of the element in the $x$ and $y$ directions, respectively, and where $\gamma(\Delta x)$ is obtained using Eq. (A6), replacing $X$ by $\Delta x$. Compute $\gamma(\Delta y)$ in a similar manner.

5. Compute $\rho$ using Eq. (A12).

6. Compute $h, w, a_{h}$, and $a_{w}$ using Eq's (17a), (17b), (13a), and (13b), respectively. When $a_{h}<1$, compute $T\left(h, a_{h}\right)$ using Eq. (14a), and when $a_{h}>1$, compute $T\left(h, a_{h}\right)$ using Eq. (14c). Similarly, compute $T\left(w, a_{w}\right)$ using Eq's (14b) and (14d), when $a_{w}<1$, and $a_{w}>1$, respectively. Solve Eq's (14a) and (14b) using 16-point Gauss quadrature.

7. Compute the probabilities of a type I $\left(p_{1}\right)$ and a type II $\left(p_{2}\right)$ error using Eq's (15) and (16), respectively.

8. If the computed probabilities of both type I and type II errors are approximately equal to or just less than the target values, then the chosen sample size is the required sample size. Otherwise, choose another sample size and repeat steps 1-7 until target probabilities are satisfied for both type I and type II errors.

\section{Application of the Proposed Method}

In order to illustrate the application of the method, an example construction cell of size $22 \mathrm{~m} \times 22$ $\mathrm{m} \times 1 \mathrm{~m}$ is considered. A construction cell size of $22 \mathrm{~m} \times 22 \mathrm{~m} \times 1 \mathrm{~m}$ (volume of $484 \mathrm{~m}^{3}$ ) is chosen for the example in this section, because 1 sample over this construction cell volume closely corresponds to the current sampling requirements specified by the USACE (2000) for cementbased S/S material (i.e., 1 sample for every $500 \mathrm{~m}^{3}$ of cement-based S/S material). The example construction cell is discretized into $320 \times 320$ elements. The hydraulic conductivity coefficient of 
variation is assumed to be 2.0 (typical for silty clay, according to Willardson and Hurst 1965) and the correlation length considered is $22 \mathrm{~m}$ (the "worst case" correlation length suggested by Liza (2014)). Since the mean is unknown prior to the QC program, the mean considered for this example is varied over values $0.1,0.9,1.0,1.1$, and 10.0 times the regulatory value. The type I and II error probabilities are computed for the number of samples of 1, 4, 9, 25, 49, and 100 using the analytical solutions presented in this paper (i.e., Eq's (15) and (16), respectively). Figures 4 and 5 show computed probabilities of type I and type II errors, respectively, for this example case. The figures indicate that for a target probability for both type I and II errors of 5\%, the QC sampling requirement for this example case is $n=25$ when the mean hydraulic conductivity is $0.9,1.0$, or 1.1 times the regulatory value (governed by the probability of a type II error, Figure 5). Thus, the USACE (2000) sampling requirement of a cement-based S/S construction cell in this example (i.e., 1 sample) is unconservative and yields greater than 5\% probability of a type II error for this example construction cell when mean hydraulic conductivities are at or close to the regulatory value (i.e., $0.9,1.0$, and 1.1 times the regulatory value) as shown in Figures 4 and 5.

\section{Using the Proposed Method to Obtain QC Sample Size: An Example}

Consider a cement-based $\mathrm{S} / \mathrm{S}$ construction cell that has a plan area of $10 \mathrm{~m} \times 10 \mathrm{~m}$. The mean hydraulic conductivity of the proposed cell is required to be less than $1 \times 10^{-8} \mathrm{~m} / \mathrm{s}$, which is the regulatory requirement. It is desired to determine the number of samples required to achieve less than a $5 \%$ probability for both type I and II errors.

Assume that the hydraulic conductivity coefficient of variation is 1.0 and that the correlation length is $3 \mathrm{~m}$ in all directions. Assume further that the actual mean hydraulic conductivity is 
$1 \times 10^{-8} \mathrm{~m} / \mathrm{s}$ under the null hypothesis, since this is the hardest case to reject. The $10 \mathrm{~m} \times 10 \mathrm{~m}$ cell is divided into $160 \times 160$ elements, each of size $0.0625 \mathrm{~m} \times 0.0625 \mathrm{~m}$. Assuming only one sample is taken from the centre of the $10 \mathrm{~m} \times 10 \mathrm{~m}$ cell, the following computations are performed.

The variance and mean of log- $k$ are as follows:

$$
\begin{aligned}
\sigma_{\ln k}^{2} & =\ln \left(1+v_{k}^{2}\right) \\
& =\ln (1+1) \\
& =0.6931 \\
\mu_{\ln k} & =\ln \mu_{k}-\frac{1}{2} \sigma_{\ln k}^{2} \\
& =\ln \left(1 \times 10^{-8}\right)-\frac{1}{2}(0.6931) \\
& =-18.7672
\end{aligned}
$$

Using $\gamma_{\ln k}(X, Y)=\gamma(X) \gamma(Y)$, where $X=Y=10 \mathrm{~m}$, and $\gamma(X)=\frac{\theta_{\ln k}^{2}}{2 X^{2}}\left[\frac{2|X|}{\theta_{\ln k}}+\exp \left\{\frac{-2|X|}{\theta_{\ln k}}\right\}-1\right]$, and similarly for $\gamma(Y)$, the variance reduction function over the cell is computed to be 0.0650 . Similarly, the variance reduction function over the element, $\gamma_{\ln k}(\Delta x, \Delta y)$, where $\Delta x=\Delta y=$ $0.0625 \mathrm{~m}$, is computed to be 0.9727 .

The mean and standard deviation of the effective hydraulic conductivity, $k_{\text {eff }}$ of the field can be computed to be,

$$
\begin{aligned}
\mu_{k_{e f f}} & =\exp \left\{\mu_{\ln k}+\frac{1}{2} \gamma_{\ln k}(X, Y) \sigma_{\ln k}^{2}\right\} \\
& =\exp \left\{-18.7672+\frac{1}{2}(0.0650)(0.6931)\right\} \\
& =7.2323 \times 10^{-9} \mathrm{~m} / \mathrm{s}
\end{aligned}
$$




$$
\begin{aligned}
\sigma_{k_{\text {eff }}} & =\sqrt{\mu_{k_{\text {eff }}}^{2}\left[\exp \left\{\sigma_{\ln k}^{2} \gamma_{\ln k}(X, Y)\right\}-1\right]} \\
& =\sqrt{\left.\left\{\left(7.2323 \times 10^{-9}\right)^{2}\right\} \exp \{(0.6931)(0.0650)\}-1\right]} \\
& =1.5532 \times 10^{-9}
\end{aligned}
$$

The standard deviation and mean of log- $k_{\text {eff }}$ can be computed to be,

$$
\begin{aligned}
\sigma_{\ln k_{e f f}} & =\sqrt{\ln \left(1+\left(\frac{\sigma_{k_{e f f}}}{\mu_{k_{e f f}}}\right)^{2}\right)} \\
& =\sqrt{\ln \left(1+\left(\frac{1.5532 \times 10^{-9}}{7.2323 \times 10^{-9}}\right)^{2}\right)} \\
= & 0.2123 \\
\mu_{\ln k_{e f f}} & \ln \left(\mu_{k_{e f f}}\right)-\frac{1}{2} \sigma_{\ln k_{e f f}^{2}} \\
= & \ln \left(7.2323 \times 10^{-9}\right)-\frac{1}{2}(0.2123)^{2} \\
& =-18.7372
\end{aligned}
$$

The mean and standard deviation of $\log -k_{G}$ can be computed as follows:

$$
\begin{gathered}
\mu_{\ln k_{G}}=\mu_{\ln k}=-18.7672 \\
\sigma_{\ln k_{G}} \cong \sqrt{\frac{1}{n^{2}\left[n\left(\sigma_{\ln k}^{2} \gamma_{\ln k}(\Delta x, \Delta y)\right)+\sum_{i=1}^{n} \sum_{\substack{j=1 \\
j \neq i}}^{n} \sigma_{\ln k}^{2} \rho_{\ln k}\left(\mathbf{x}_{i}-\mathbf{x}_{j}\right)\right]}} \\
=\sqrt{\frac{1}{1^{2}}[(1)(0.6931)(0.9660)+0]}
\end{gathered}
$$




$$
=0.8211
$$

Since only one sample is taken from the cell, the variance reduction is only for averaging within the sample.

Using

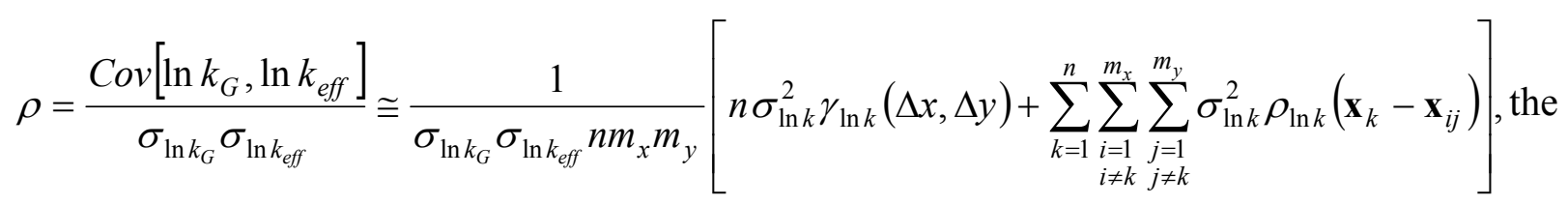

correlation coefficient between $\ln k_{e f f}$ and $\ln k_{G}$ can be computed to be 0.3328 .

$h, w, a_{h}$, and $a_{w}$ are computed as follows:

$$
\begin{aligned}
h & =\frac{\ln \left(1 \times 10^{-8}\right)-\mu_{\ln k_{G}}}{\sigma_{\ln k_{G}}} \\
& =0.4220 \\
w & =\frac{\ln \left(1 \times 10^{-8}\right)-\mu_{\ln k_{e f f}}}{\sigma_{\ln k_{e f f}}} \\
& =1.6320 \\
a_{h} & =\frac{w}{h \sqrt{1-\rho^{2}}}-\frac{\rho}{\sqrt{1-\rho^{2}}} \\
& =3.7476 \\
a_{w} & =\frac{h}{w \sqrt{1-\rho^{2}}}-\frac{\rho}{\sqrt{1-\rho^{2}}} \\
& =-0.0786
\end{aligned}
$$

Since $a_{h}>1, T\left(h, a_{h}\right)$ can be computed using 
$T\left(h, a_{h}\right)=\frac{1}{2} \Phi(h)+\frac{1}{2} \Phi\left(h a_{h}\right)-\Phi(h) \Phi\left(h a_{h}\right)-T\left(h a_{h}, \frac{1}{a_{h}}\right)=0.1659$

where

$T\left(h a_{h}, \frac{1}{a_{h}}\right)=\frac{1}{2 \pi} \int_{0}^{1 / a_{h}} \frac{\exp \left[-\frac{1}{2}\left(h a_{h}\right)^{2}\left(1+u^{2}\right)\right]}{1+u^{2}} d u$, is computed using 16-point Gauss quadrature to be 0.0115 .

Similarly, using 16-point Gauss quadrature, $T\left(w, a_{w}\right)=\frac{1}{2 \pi} \int_{0}^{a_{w}} \frac{\exp \left[-\frac{1}{2} w^{2}\left(1+v^{2}\right)\right]}{1+v^{2}} d v$ is computed to be -0.0033 .

The probabilities of a type I $\left(p_{1}\right)$ and a type $\mathrm{II}\left(p_{2}\right)$ error are thus computed to be,

$$
\begin{aligned}
p_{1} & =\frac{1}{2} \Phi(h)-\frac{1}{2} \Phi(w)+T\left(h, a_{h}\right)+T\left(w, a_{w}\right) \\
& =\frac{1}{2} \Phi(0.4220)-\frac{1}{2} \Phi(1.6320)+0.1659-0.0033 \\
& =0.0201 \\
p_{2} & =\frac{1}{2} \Phi(w)-\frac{1}{2} \Phi(h)+T\left(h, a_{h}\right)+T\left(w, a_{w}\right) \\
& =\frac{1}{2} \Phi(1.6320)-\frac{1}{2} \Phi(0.4220)+0.1659-0.0033 \\
& =0.3052
\end{aligned}
$$

Since the computed probability of a type II error $(30.52 \%)$ is greater than the target value $(5 \%)$, the sample size of $n=1$ is not acceptable. The probabilities of type I and II errors are further computed for $n=4,9,16,25$, and 49 , where the samples are located at equal spacing in both of the $x$ and $y$ directions, in Table 2. Table 2 shows that both type I and II error probabilities are 
less than $5 \%$ when the number of samples is 49 , implying that $n=49$ is the required number of samples for this example case.

\section{Summary}

In this paper, an analytical approach is proposed to estimate the sample size, for the QC program of a cement-based $\mathrm{S} / \mathrm{S}$ construction cell, required to achieve target type I and II error probabilities for the hypothesis test considered in this study. The analytical solutions developed are functions of the number of samples taken and the statistics of the hydraulic conductivity field. For a range of parameter sets, the analytically computed probabilities of type I and II errors are compared to those estimated via probabilistic simulations with excellent agreement, allowing the probabilities of a type I and a type II error to be computed analytically with reasonable confidence and used to develop rational sampling requirements for the QC program of construction cell. An example has been presented to illustrate how the proposed method can be used in practice to assess required QC sample size of cement-based S/S construction cell.

\section{Acknowledgements}

The authors gratefully acknowledge the financial support of the Natural Sciences and Engineering Research Council of Canada and its STEWARD program.

\section{References}

Fenton, G.A., and Griffiths, D. V. 1993. Statistics of block conductivity through a simple bounded stochastic medium. Water Resources Research, 29(6): 1825-1830.

Fenton, G.A. 1999. Estimation for stochastic soil models. ASCE Journal of Geotechnical and Geoenvironmental Engineering, 125(6): 470-485. 
Fenton, G.A., and Griffiths, D.V. 2008. Risk Assessment in Geotechnical Engineering. John Wiley \& Sons, New York.

Fenton, G.A., Liza, R., Lake, C.B., Menzies, W. T., and Griffiths, D. V. 2015. Statistical sample size for quality control programs of cement-based solidification/stabilization. Canadian Geotechnical Journal, 52(10): 1620-1628.

Hills, C., Bates, E., and Gunning, P. 2015. Stabilisation and Solidification of Contaminated Soil and Waste: Science and Practice. Hygge Media, UK.

Liza, R. 2014. Statistical sample size for quality control programs of cement-based solidification/stabilization. Ph. D. Thesis, Dalhousie University, Halifax, NS, Canada.

Owen, D.B. 1959. Tables for computing bivariate normal probabilities. The Annals of Mathematical Statistics, 27(4): 1075-1090.

USACE. 2000. Solidification/Stabilization (S/S) of contaminated material. Section 02160 A, Washington, D.C.

Willardson, L.S., and Hurst, R.L. 1965. Sample size estimates in permeability studies. J. Irrig. Drain. Div. Amer. Soc. Civil Eng., 91(IRI): 1-9. 


\section{List of Symbols}

The following symbols are used in this paper:

$A=$ Construction cell area perpendicular to flow i.e., $Y \times 1$

$h=$ Standardization of $k_{\text {crit }}$ with respect to $k_{G}$

$H_{o} \quad=$ Null hypothesis

$H_{a} \quad=$ Alternative hypothesis

$i \quad=$ Hydraulic gradient in the $x$ direction of the construction cell

$k_{\text {crit }}=$ Regulatory hydraulic conductivity

$k_{\text {eff }} \quad=$ Effective hydraulic conductivity

$k_{G}=$ Estimated effective hydraulic conductivity

$k_{i} \quad=$ Hydraulic conductivity of the $i$ th element

$k_{j} \quad=$ Hydraulic conductivity of the $j$ th sample

$k \quad=$ Hydraulic conductivity field

$\ln k=$ Log-hydraulic conductivity field

$\ln k_{c r i t}=$ Log-regulatory hydraulic conductivity

$\ln k_{\text {eff }} \quad=$ Log-effective hydraulic conductivity

$\ln k_{G} \quad=$ Log-estimated effective hydraulic conductivity

$m \quad=$ Number of elements in the random field

$m_{x} \quad=$ Number of elements in the $x$ direction of the random field

$m_{y} \quad=$ Number of elements in the $y$ direction of the random field

$n \quad=$ Number of samples

$p_{1} \quad=$ Probability of a type I error

$p_{2} \quad=$ Probability of a type II error

$Q \quad=$ Total flow through the construction cell

$r \quad=$ Dummy variable of integration

$s \quad=$ Dummy variable of integration 
$w \quad=$ Standardization of $k_{c r i t}$ with respect to $k_{e f f}$

$X=$ Planar dimension of the construction cell in the $x$ direction

$\Delta x=$ Planar dimension of the element in the $x$ direction

$Y \quad=$ Planar dimension of the construction cell in the $y$ direction

$\Delta y=$ Planar dimension of the element in the $y$ direction

$\theta_{\ln k}=$ Correlation length of the $\ln k$ random field

$\mu_{k} \quad=$ Mean of the hydraulic conductivity field

$\mu_{k}^{\prime} \quad=$ Normalized mean of the hydraulic conductivity field

$\mu_{\ln k}=$ Mean of the log-hydraulic conductivity field, $\ln k$

$\mu_{\ln k_{G}}=$ Mean of the log-estimated effective hydraulic conductivity, $\ln k_{G}$

$\mu_{\ln k_{e f f}}=$ Mean of the log-effective hydraulic conductivity, $\ln k_{\text {eff }}$

$\sigma_{\ln k}=$ Standard deviation of the log-hydraulic conductivity field, $\ln k$

$\sigma_{\ln k_{G}}=$ Standard deviation of the log-estimated effective hydraulic conductivity, $\ln k_{G}$

$\sigma_{\ln k_{e f f}}=$ Standard deviation of the log-effective hydraulic conductivity, $\ln k_{\text {eff }}$

$\sigma_{k} \quad=$ Standard deviation of the hydraulic conductivity field

$v_{k} \quad=$ Coefficient of variation of the hydraulic conductivity field

$\rho \quad=$ Correlation coefficient between $\ln k_{G}$ and $\ln k_{\text {eff }}$

$\rho_{\ln k}=$ Correlation coefficient between two points in the $\ln k$ random field

$\gamma_{\ln k}=$ Variance reduction function when $\ln k$ is averaged over some volume

$\gamma \quad=$ Same as $\gamma_{\ln k}$

$\tau=$ Distance between two points in the random field

$\Phi=$ Cumulative distribution function of the standard normal variate 


\section{Figure Captions}

Figure 1: The two-dimensional random hydraulic conductivity field used in this paper Figure 2: Comparison between the theory and simulation for the probability of a type I error

Figure 3: Comparison between the theory and simulation for the probability of a type II error

Figure 4: Probability of a type I error for the example case

Figure 5: Probability of a type II error for the example case 


\section{Appendix A}

\section{Statistics of a Geometric Average}

Assuming a Markovian correlation structure (Vanmarcke 1984) with a separable correlation function (a product of directional correlation functions) and correspondingly a separable variance reduction function, the mean and standard deviation of the effective hydraulic conductivity of the $\mathrm{S} / \mathrm{S}$ construction cell, $k_{\text {eff }}$, can be calculated as,

$$
\begin{aligned}
& \mu_{k_{e f f}}=\exp \left\{\mu_{\ln k}+\frac{1}{2} \gamma_{\ln k}(X, Y) \sigma_{\ln k}^{2}\right\} \\
& \sigma_{k_{e f f}}=\sqrt{\mu_{k_{e f f}}^{2}\left[\exp \left\{\sigma_{\ln k}^{2} \gamma_{\ln k}(X, Y)\right\}-1\right]}
\end{aligned}
$$

where

$$
\begin{gathered}
\mu_{\ln k}=\ln \mu_{k}-\frac{1}{2} \sigma_{\ln k}^{2} \\
\sigma_{\ln k}^{2}=\ln \left(1+v_{k}^{2}\right) \\
\gamma_{\ln k}(X, Y)=\gamma(X) \gamma(Y) \\
\gamma(X)=\frac{\theta_{\ln k}^{2}}{2 X^{2}}\left[\frac{2|X|}{\theta_{\ln k}}+\exp \left\{-\frac{2|X|}{\theta_{\ln k}}\right\}-1\right]
\end{gathered}
$$

and similarly for $\gamma(Y)$ and $v_{k}=\frac{\sigma_{k}}{\mu_{k}}$ is the coefficient of variation of point-scale hydraulic conductivity.

The mean and standard deviation of $\ln k_{\text {eff }}$ can be computed as,

$$
\mu_{\ln k_{e f f}}=\ln \left(\mu_{k_{e f f}}\right)-\frac{1}{2} \sigma_{\ln k_{e f f}}^{2}
$$




$$
\sigma_{\ln k_{e f f}}=\sqrt{\ln \left(1+v_{k_{e f f}}^{2}\right)}
$$

where $v_{k_{e f f}}=\frac{\sigma_{k_{e f f}}}{\mu_{k_{e f f}}}$ is the coefficient of variation of the effective hydraulic conductivity.

Assuming $k_{G}$ to be the geometric average of $n$ sample hydraulic conductivities, the mean and standard deviation of the logarithm of the estimated effective hydraulic conductivity, $k_{G}$, can be calculated as,

$$
\mu_{\ln k_{G}}=\mu_{\ln k}
$$

and

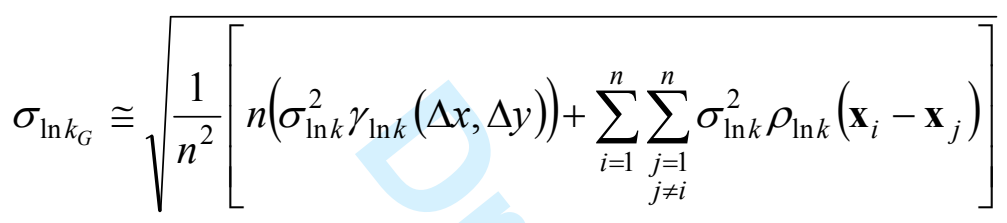

where $\Delta x$ and $\Delta y$ are the averaging dimensions of the sample and $\mathbf{x}_{i}=\left\{x_{i x}, x_{i y}\right\}$ are the spatial coordinate of the center of the $i$ th sample. A Markovian correlation structure with a separable correlation function (which is a product of directional correlation functions) and isotropic correlation lengths is assumed here, as follows,

$$
\rho_{\ln k}\left(\mathbf{x}_{i}-\mathbf{x}_{j}\right)=\exp \left\{-2\left|x_{i x}-x_{j x}\right| / \theta_{\ln k}\right\} \exp \left\{-2\left|x_{i y}-x_{j y}\right| / \theta_{\ln k}\right\}
$$

Note that Eq. A10 is an approximation, as is Eq. A 12 below, since the correlation coefficient between local averages has been approximated by the correlation coefficient between the centers of the samples in Eq. A 11. However, for values of $\Delta x$ and $\Delta y$ small relative to the correlation length, the approximation is quite accurate.

The correlation coefficient between $\ln k_{\text {eff }}$ and $\ln k_{G}, \rho$ is given by, 


$$
\rho=\frac{\operatorname{Cov}\left\lfloor\ln k_{G}, \ln k_{e f f}\right\rfloor}{\sigma_{\ln k_{G}} \sigma_{\ln k_{e f f}}}
$$

[A12]

$$
\cong \frac{1}{\sigma_{\ln k_{G}} \sigma_{\ln k_{e f f}} n m_{x} m_{y}}\left[n \sigma_{\ln k}^{2} \gamma_{\ln k}(\Delta x, \Delta y)+\sum_{k=1}^{n} \sum_{\substack{i=1 \\ i \neq k}}^{m_{x}} \sum_{\substack{j=1 \\ j \neq k}}^{m_{y}} \sigma_{\ln k}^{2} \rho_{\ln k}\left(\mathbf{x}_{k}-\mathbf{x}_{i j}\right)\right]
$$

where $m_{x}$ and $m_{y}$ are the number of elements in the $x$ and $y$ directions, respectively, such that $m_{x} \times m_{y}=m$ where $m$ is the number of elements in the random field.

\section{Appendix B}

\section{Derivations of Error Probabilities Using Owen's (1959) Approximation}

Let $f_{\ln k_{e f f} \ln k_{G}}(r, s)$ be the bivariate normal probability density function of random variables $\ln k_{e f f}$ and $\ln k_{G}, f_{\ln k_{G}}(s)$ be the marginal probability density function of $\ln k_{G}, u=\frac{r-\mu_{\ln k_{e f f}}}{\sigma_{\ln k_{e f f}}}$, $v=\frac{s-\mu_{\ln k_{G}}}{\sigma_{\ln k_{G}}}$, and $B(h, w ; \rho)$, where $h=\frac{\ln k_{c r i t}-\mu_{\ln k_{G}}}{\sigma_{\ln k_{G}}}, w=\frac{\ln k_{c r i t}-\mu_{\ln k_{e f f}}}{\sigma_{\ln k_{e f f}}}$, be Owen's (1959) approximation to the bivariate normal probability (see Eq's 11 and 12).

Then, the probability of a type I error, $\left.p_{1}=\mathrm{P} \mid \ln k_{G}<\ln k_{c r i t} \cap \ln k_{\text {eff }}>\ln k_{\text {crit }}\right\rfloor$, is,

$$
\begin{aligned}
p_{1} & =\int_{-\infty}^{\ln k_{c r i t}} \int_{\ln k_{\text {crit }}}^{+\infty} f_{\ln k_{\text {eff }} \ln k_{G}}(r, s) d r d s \\
& =\int_{-\infty}^{\ln k_{\text {crit }}}\left\{f_{\ln k_{G}}(s)-\int_{-\infty}^{\ln k_{\text {crit }}} f_{\ln k_{e f f} \ln k_{G}}(r, s) d r\right\} d s
\end{aligned}
$$




$$
\begin{aligned}
& =\Phi\left(\frac{\ln k_{c r i t}-\mu_{\ln k_{G}}}{\sigma_{\ln k_{G}}}\right)-\int_{-\infty}^{\ln k_{c r i t} \ln k_{c r i t}} \int_{-\infty} f_{\ln k_{e f f} \ln k_{G}}(r, s) d r d s \\
& =\Phi\left(\frac{\ln k_{c r i t}-\mu_{\ln k_{G}}}{\sigma_{\ln k_{G}}}\right)-\int_{-\infty}^{\ln k_{c r i t} \ln k_{\text {crit }}} \int_{-\infty}\left[\frac{1}{2 \pi \sigma_{\ln k_{e f f}} \sigma_{\ln k_{G}} \sqrt{1-\rho^{2}}} \exp \left\{-\frac{1}{2\left(1-\rho^{2}\right)}\left(u^{2}-2 \rho u v+v^{2}\right)\right\}\right]
\end{aligned}
$$

$d r d s$

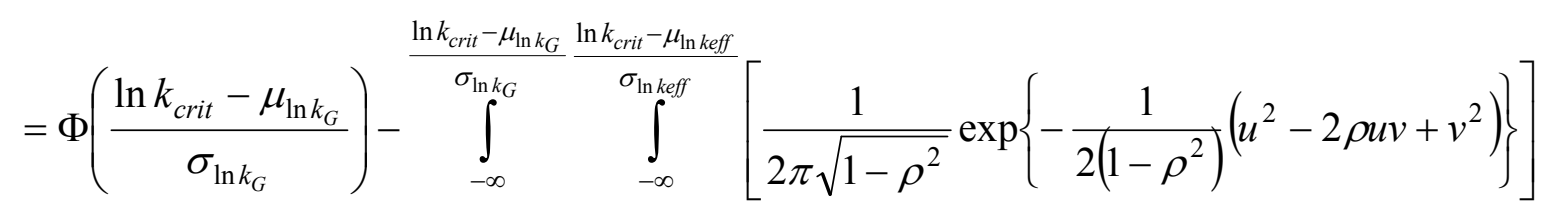

$d u d v$

$$
\begin{aligned}
& =\Phi(h)-\int_{-\infty}^{h} \int_{-\infty}^{w}\left[\frac{1}{2 \pi \sqrt{1-\rho^{2}}} \exp \left\{-\frac{1}{2\left(1-\rho^{2}\right)}\left(u^{2}-2 \rho u v+v^{2}\right)\right\}\right] d u d v \\
& =\Phi(h)-B(h, w, \rho) \\
& =\Phi(h)-\left[\frac{1}{2} \Phi(h)+\frac{1}{2} \Phi(w)-T\left(h, a_{h}\right)-T\left(w, a_{w}\right)\right] \\
& {[\mathrm{B} 1] \quad=\frac{1}{2} \Phi(h)-\frac{1}{2} \Phi(w)+T\left(h, a_{h}\right)+T\left(w, a_{w}\right)}
\end{aligned}
$$

In a similar manner, the probability of a type II error, $p_{2}=\mathrm{P}\left\lfloor\ln k_{G}>\ln k_{\text {crit }} \cap \ln k_{\text {eff }}<\ln k_{\text {crit }}\right\rfloor$ can be derived to be

$$
p_{2}=\frac{1}{2} \Phi(w)-\frac{1}{2} \Phi(h)+T\left(h, a_{h}\right)+T\left(w, a_{w}\right)
$$

The above expressions for the probabilities of type I and type II errors are valid if $h w>0$ or if $h w=0$ and $h$ or $w \geq 0$. If $h w<0$ or if $h w=0$ and $h$ or $w<0$, the probabilities of type I and type II errors are as follows 
[B3]

$$
\begin{aligned}
& p_{1}=\frac{1}{2} \Phi(h)-\frac{1}{2} \Phi(w)+T\left(h, a_{h}\right)+T\left(w, a_{w}\right)+\frac{1}{2} \\
& p_{2}=\frac{1}{2} \Phi(w)-\frac{1}{2} \Phi(h)+\mathrm{T}\left(h, a_{h}\right)+\mathrm{T}\left(w, a_{w}\right)+\frac{1}{2}
\end{aligned}
$$

\section{References}

Vanmarcke, E.H. 1984. Random Fields: Analysis and Synthesis. The MIT press, Cambridge, Massachusetts, USA.

\section{List of Symbols}

The following symbols are used in Appendices:

$h \quad=$ Standardization of $k_{\text {crit }}$ with respect to $k_{G}$

$k_{\text {eff }} \quad=$ Effective hydraulic conductivity

$k_{G} \quad=$ Estimated effective hydraulic conductivity

$\ln k_{c r i t}=$ Log-regulatory hydraulic conductivity

$\ln k_{\text {eff }} \quad=$ Log-effective hydraulic conductivity

$\ln k_{G} \quad=$ Log-estimated effective hydraulic conductivity

$m \quad=$ Number of elements in the random field

$m_{x} \quad=$ Number of elements in the $x$ direction of the random field

$m_{y} \quad=$ Number of elements in the $y$ direction of the random field

$n \quad=$ Number of samples

$p_{1} \quad=$ Probability of a type I error

$p_{2} \quad=$ Probability of a type II error

$r \quad=$ Dummy variable of integration

$s \quad=$ Dummy variable of integration

$w \quad=$ Standardization of $k_{\text {crit }}$ with respect to $k_{\text {eff }}$ 
$X=$ Planar dimension of the construction cell in the $x$ direction

$\Delta x=$ Planar dimension of the element in the $x$ direction

$Y \quad=$ Planar dimension of the construction cell in the $y$ direction

$\Delta y=$ Planar dimension of the element in the $y$ direction

$\theta_{\ln k}=$ Correlation length of the $\ln k$ random field

$\mu_{k} \quad=$ Mean of the hydraulic conductivity field

$\mu_{k_{e f f}}=$ Mean of the effective hydraulic conductivity

$\mu_{\ln k}=$ Mean of the log-hydraulic conductivity field, $\ln k$

$\mu_{\ln k_{G}}=$ Mean of the log-estimated effective hydraulic conductivity, $\ln k_{G}$

$\mu_{\ln k_{\text {eff }}}=$ Mean of the log-effective hydraulic conductivity, $\ln k_{\text {eff }}$

$\sigma_{k} \quad=$ Standard deviation of the hydraulic conductivity field

$\sigma_{k_{e f f}}=$ Standard deviation of the effective hydraulic conductivity

$\sigma_{\ln k}=$ Standard deviation of the log-hydraulic conductivity field, $\ln k$

$\sigma_{\ln k_{G}}=$ Standard deviation of the log-estimated effective hydraulic conductivity, $\ln k_{G}$

$\sigma_{\ln k_{e f f}}=$ Standard deviation of the log-effective hydraulic conductivity, $\ln k_{\text {eff }}$

$v_{k} \quad=$ Coefficient of variation of the hydraulic conductivity field

$v_{k_{e f f}}=$ Coefficient of variation of the effective hydraulic conductivity

$\rho \quad=$ Correlation coefficient between $\ln k_{G}$ and $\ln k_{\text {eff }}$

$\rho_{\ln k}=$ Correlation coefficient between two points in the $\ln k$ random field

$\gamma_{\ln k}=$ Variance reduction function when $\ln k$ is averaged over some volume

$\gamma \quad=$ Same as $\gamma_{\ln k}$

$\Phi=$ Cumulative distribution function of the standard normal variate 


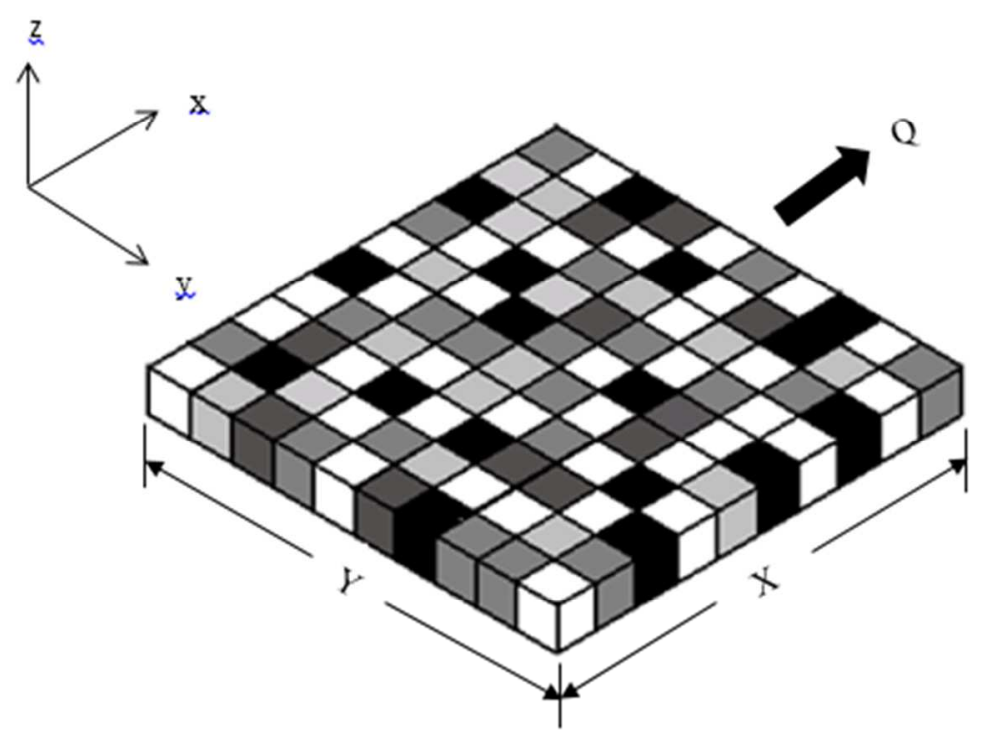

Figure 1: The two-dimensional random hydraulic conductivity field used in this paper

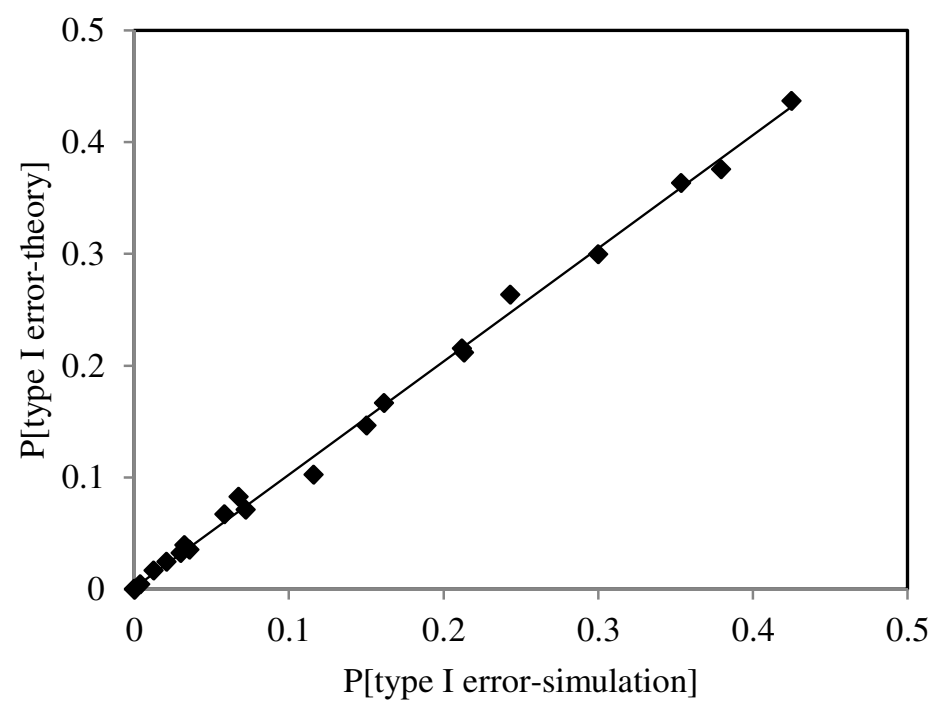

Figure 2: Comparison between the theory and simulation for the probability of a type I error 


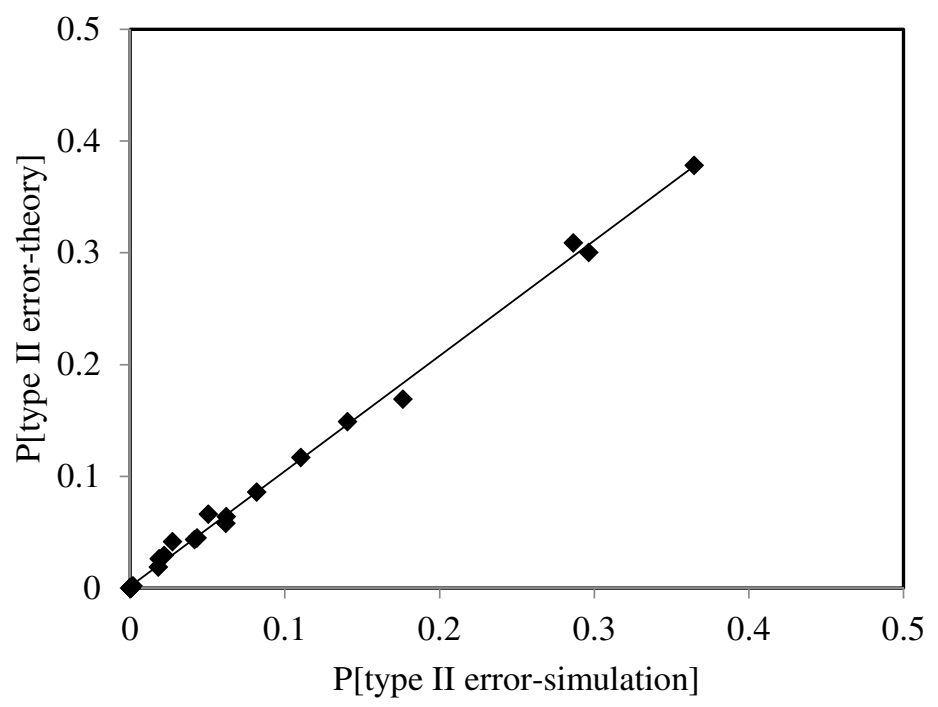

Figure 3: Comparison between the theory and simulation for the probability of a type II error

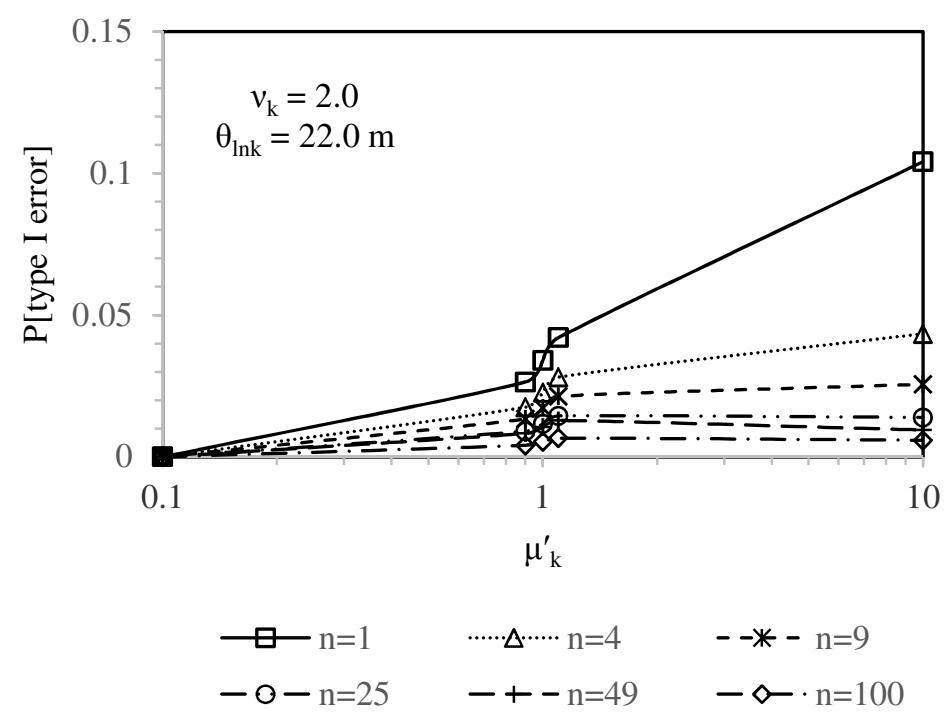

Figure 4: Probability of a type I error for the example case 


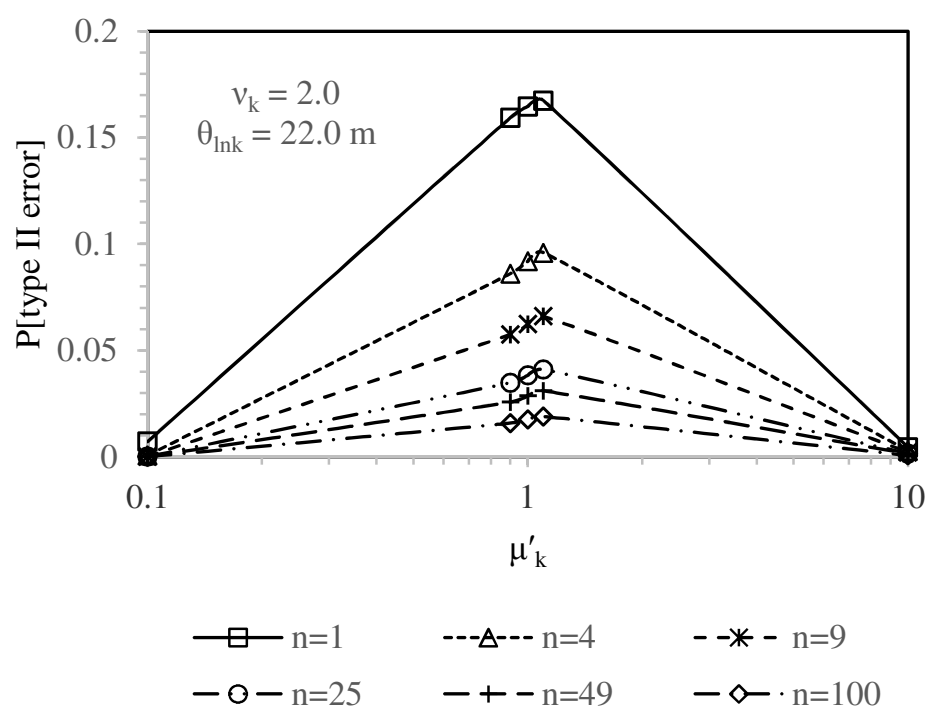

Figure 5: Probability of a type II error for the example case 
Table 1: Parametric variations considered in the simulations

\begin{tabular}{|l|l|}
\hline Parameter point-mean hydraulic & $0.5,1.0,1.2$, and 1.5. \\
\hline Normalized $\quad$ Variation \\
\hline Coefficient of variation, $v_{k}=\sigma_{k} / \mu_{k}$ & \\
\hline Correlation length, $\theta_{\ln k}$ & $0.5,1.0$, and 2.0. \\
\hline Number of samples, $n$ & $1 \mathrm{~m}, 3 \mathrm{~m}$, and $10 \mathrm{~m}$. \\
\hline
\end{tabular}

Table 2: The probabilities of type I $\left(p_{1}\right)$ and type II $\left(p_{2}\right)$ errors for $\mu_{k}=1 \times 10^{-8} \mathrm{~m} / \mathrm{s}, v_{k}=1.0$, $\theta_{k}=3 \mathrm{~m}$, and varying $n$

\begin{tabular}{|c|c|c|}
\hline \multirow{2}{*}{$n$} & \multicolumn{2}{|c|}{$\theta_{k}=3 \mathrm{~m}$} \\
\cline { 2 - 3 } & $p_{1}$ & $p_{2}$ \\
\hline 1 & 0.0201 & 0.3052 \\
\hline 4 & 0.0162 & 0.1889 \\
\hline 9 & 0.0136 & 0.1240 \\
\hline 16 & 0.0119 & 0.0883 \\
\hline 25 & 0.0095 & 0.0640 \\
\hline 49 & 0.0080 & 0.0437 \\
\hline
\end{tabular}

